\title{
¿Tiene precio el éxito académico? La relación entre inversión educativa y resultados escolares
}

\author{
Jessica Menéndez Lastra* y Rubén Fernández-Alonso
}

Universidad de Oviedo

\author{
PALABRAS CLAVE \\ Inversión educativa \\ Economía de la educación \\ Recursos educativos \\ Gasto educativo \\ Resultados educativos
}

\author{
KEYWORDS \\ Investment in education \\ Economics of education \\ School resources \\ School expenditures \\ Educational outcomes
}

\begin{abstract}
RESUMEN
El incremento de la inversión y los recursos es una demanda recurrente para la mejora del sistema educativo, y es habitual que gestores, docentes y familias señalen la escasez de recursos como una causa de los bajos resultados escolares. La presente investigación explora las relaciones entre los resultados escolares y el gasto e inversión educativa. Para ello se establecen tres objetivos: comparar la situación de las comunidades autónomas (CCAA) en los indicadores riqueza, inversión educativa y resultados educativos; analizar la relación entre dichos indicadores; y, comprobar la capacidad de los indicadores de riqueza y recursos para predecir los resultados en PISA 2015. Para lograr el primer objetivo se calcularon estadísticos descriptivos y análisis de frecuencias. Para el segundo objetivo se realizó un análisis de correlaciones de Pearson y para el tercer objetivo se ajustaron dos modelos de regresión múltiple. Las principales conclusiones son las siguientes: las CCAA difieren en los indicadores de riqueza, recursos y resultados educativos; la relación entre los indicadores de recursos y resultados es baja o muy baja; la única variable con cierta capacidad predictiva de los resultados de las administraciones educativas PISA es su nivel de riqueza. Los datos corroboran las evidencias de investigaciones previas: España ha alcanzado un nivel de riqueza e inversión educativa lo suficientemente alto para que en la relación inversión-resultados el cómo (gestión de los recursos) sea más importante que el cuánto (recursos disponibles).
\end{abstract}

\section{Does academic success have a price? The relationship between educa- tional investment and school results}

\begin{abstract}
The increase in investment and resources in education is a recurring demand for the improvement of the educational system. It is usual for school leaders, teachers and families to point out the scarcity of resources as a cause for a low achievement. This research explores the relations between achievement and spending and investment in education. This study aims to three objectives: it compares the current situation of Spanish regions regarding variables of resources and outcomes; it analyses the relation among wealth, investment and resources variables; and finally, it explores the prediction ability of the wealth and resources variables over the standardized tests results. To accomplish
\end{abstract}

Universidad de Oviedo

Autora de correspondencia: * Jessica Menéndez Lastra; jessmlastra@gmail.com

Recibido: 09/11/2020 - Aceptado: 17/02/2021

Revista de Formación del Profesorado e Investigación Educativa

Facultad de Formación del Profesorado y Educación

Universidad de Oviedo

Enero - Diciembre 2021

ISSN: $2340-4728$

(c) (1) $\circledast)$ Esta obra está bajo una licencia internacional Creative Commons

Esta obra está bajo una licencia internacional
Atribución-NoComercial-SinDerivadas 4.0. 
the first aim, descriptive statistics and frequency analysis were calculated. For the second one, a Pearson correlation coefficient was calculated. Finally, for the third aim, two multiple regression models were performed in which the variables of interest were the results in PISA 2015. The main conclusions of this research are as follows: the variables of wealth, resources and outcomes differs among Spanish regions, the relation between resources and results variables is low or very low, the only variable which shows some predictive ability over the PISA standardized test results is the GDP per capita. The data supports the idea that Spain is a wealthy country with a high investment in education which should be enough to confirm that the resource management should be more important than the amount of money spent in education.

\section{Introducción}

La educación tiene un extraordinario potencial para el desarrollo de los países, y sus sistemas educativos deben proporcionar los recursos que garanticen oportunidades educativas de calidad (United Nations, 2015). Ello plantea serios interrogantes al gestor educativo: ¿cuántos recursos son necesarios?, ¿qué efectos produce la inversión educativa? Para responder a los mismos la investigación implementa modelos de análisis que predicen los resultados educativos a partir de la riqueza nacional y el volumen de inversión, el gasto por estudiante, el salario docente y la ratio alumnado/profesorado (Hanushek, 1986, 1997).

Las conclusiones de la investigación que relaciona inversión y resultados educativos parecen dependientes del contexto. Los estudios realizados en países en vías de desarrollo señalan que el aumento de la inversión pública en entornos desfavorecidos incrementa las tasas de escolarización, mejora significativamente los resultados escolares y reduce las desigualdades educativas (Abott et al., 2020; Candelaria y Shores, 2019; Cunningham et al., 2019; Gaviria et al., 2004; Harbison y Hanushek, 1992; Lafortune et al., 2018; Vegas y Coffin, 2015). Sin embargo, en países desarrollados las conclusiones no son tan diáfanas (Glewwe et al., 2015; Glewwe y Lambert, 2010). Hanushek (1986, 1997), después de compendiar la investigación de la segunda mitad del siglo XX, afirma que "no parece existir una relación fuerte o sistemática entre el gasto educativo y los desempeños escolares" (Hanushek, 1986, p. 1162). La Organisation for Economic Co-operation and Development ([OECD], 2012) ha establecido un límite para interpretar los efectos de la inversión educativa: observa que cuando el Producto Interior Bruto (PIB) per cápita de los países supera los 20.000 \$ medidos en Paridad de Poder Adquisitivo (\$-PPA) el gasto total deja de ser un factor predictivo de los resultados en PISA; y concluye que, a partir de este valor lo que se gasta en educación es menos importante que cómo se emplean esos recursos.

El gasto por estudiante es otra variable muy analizada, especialmente en el último tercio del siglo $\mathrm{XX}$, cuando este concepto de gasto experimentó un fuerte incrementó a nivel mundial (Hanushek et al., 2019). No obstante, la investigación educativa no encontró un efecto directo y positivo entre el aumento del gasto por estudiante y los logros académicos (Arroyo-Resino et al., 2019; Jackson et al., 2016; Vegas y Coffin, 2015). Jackson et al. (2016) aluden a la dificultad analítica de separar el efecto neto de este indicador del impacto de las variables del contexto familiar y escolar, que suelen tener bastante potencia explicativa (Fernández-Alonso y Muñiz, 2019). Sin embargo, más bien parece que, como ocurre con otros factores de recursos, el impacto del gasto por estudiante solo es apreciable hasta cierta cantidad. Así, la OECD (2012) estima que, cuando la inversión acumulada por estudiante entre los 6 y los 15 años supera los 35.000 \$-PPA la relación gasto por estudiante-resultados se diluye; y Vegas y Coffin (2015), tomando el gasto anualizado, observan que la relación desaparece al superar los 8.000 \$-PPA por estudiante y año. En línea similar, Pritchett y Filmer (1999) concluyen que en el gasto por estudiante el cómo importa más que el cuánto.

La investigación sobre ingresos e incentivos salariales del docente presenta igualmente resultados poco concluyes. Los estudios de comparación salarial entre países advierten que la calidad de un sistema educativo no puede ser superior al nivel de sus docentes (Coffield, 2012), o directamente afirman que la calidad del profesorado no está relacionada con su salario (Hanushek et al., 2019). En definitiva, parece que la enseñanza eficiente depende más de la motivación y disposición del docente que de los incentivos salariales (Pritchett y Filmer, 1999). No obstante, los estudios que analizan las diferencias salariales entre provincias de un mismo país apuntan a que las variaciones pueden hacer más atractivas a determinadas regiones para el profesorado más cualificado, produciendo así mejores resultados en las provincias con mayores salarios (Leclercq, 2005). En todo caso, las políticas que vinculan incentivos salariales y resultados educativos presentan riesgos importantes: Hanushek y Woessmann (2011) documentaron cómo la ligazón entre primas salariales y calificaciones académicas en Kenia acabó derivando en la manipulación de los exámenes.

Finalmente, con respecto a la ratio estudiantes/docente existen dos estrategias de gestión diferenciadas: países que sostienen que reducir el tamaño de la clase tiene efectos muy modestos, existiendo otras estrategias menos costosas para mejorar los estándares académicos, y países con políticas orientadas a reducir las ratios aunque ello incremente la inversión (Krueger, 1999; Rivkin et al., 2005). La conclusión general es que la relación entre reducción de ratios y mejoras de resultados es débil y en ocasiones inexistente (Leuven y Oosterbeek, 2018; OECD, 2016a). Incluso cuando esta reducción se ha centrado en grupos más desaventajados las ganancias han sido escasas, probablemente porque la reducción del tamaño del aula se focaliza en los grupos más desfavorecidos, lo que no es suficiente para mejorar el rendimiento del conjunto del sistema (Filges et al., 2018; Molnar et al., 2001; Zyngier, 2014). Por tanto, aunque teóricamente las aulas pequeñas son una buena base para incrementar los resultados escolares en absoluto garantizan el éxito académico, existiendo evidencias de que los objetivos también se pueden alcanzar en grupos mayores (Filges et al., 2018; Molnar et al., 2001; Schwerdt y Woessmann, 2020).

\section{Objetivos}

Esta investigación analiza la relación entre recursos y los resultados educativos en las comunidades autónomas españolas (CCAA), planteándose los siguientes objetivos específicos:

1. Describir y comparar las diferencias entre las CCAA en cuanto a riqueza (PIB per cápita), inversión educativa (gasto por estudiante, salario docente y ratio estudiantes/docente) y resultados educativos (promedios en PISA 2015 y tasa de idoneidad a los 15 años).

2. Analizar la relación existente entre los indicadores de riqueza, inversión y resultados en el conjunto de las CCAA. 
3. Comprobar la capacidad de los indicadores de riqueza y recursos para predecir los resultados en PISA de las CCAA.

\section{Metodología}

\section{Muestra}

Se analizaron los indicadores de las 17 CCAA y de las ciudades autónomas de Ceuta y Melilla, cuando estas disponían de datos propios y segregados.

\section{Instrumentos y variables}

- $\quad$ Riqueza. Se expresa como el PIB per cápita del año 2015 publicado en la página web del Instituto Nacional de Estadística.

- Gasto público por estudiante. Se define como el gasto público por alumno de educación no universitaria en el curso 2014/15. Los datos provienen del Sistema Estatal de Indicadores de la Educación (SEIE) del Ministerio de Educación y Formación Profesional.

- Salario docente. Son los ingresos brutos anuales en el año 2015 del profesorado de enseñanza secundaria en centros públicos, con seis años de antigüedad en la carrera docente y sin complementos retributivos singulares. Las estadísticas oficiales no ofrecen este dato segregado por administración educativa, por lo que es de elaboración propia. Se calculó como la suma de las retribuciones anuales básicas (salario base y trienios por antigüedad establecidos por la Ley de Presupuestos Generales del Estado) y complementarias, cuya cuantía depende de la legislación autonómica.

- Ratio o proporción estudiantes por docente. Se expresa como el número medio de estudiantes por profesor equivalente a tiempo completo en centros de enseñanza de régimen general en el curso 2014/15. La fuente fue la "Educabase" del Ministerio de Educación y Formación Profesional.

- Tasa de idoneidad a los 15 años. Es la proporción de estudiantes de 15 años que están matriculados en el curso correspondiente a su edad ( $4^{\circ}$ ESO) y fue extraída del SEIE.

- Resultados en PISA 2015. Es el promedio en Lectura, Matemáticas y Ciencias en el Programa para la Evaluación Internacional de Estudiantes (PISA) en el año 2015, que fue recuperado de la plataforma "PISA Data Explorer".

\section{Procedimiento}

Como se acaba de mencionar los datos provienen de fuentes oficiales. Además, para contextualizar algunos resultados, se consultaron las estadísticas e indicadores de la OECD (2016a). Adicionalmente, se extrajo el número de estudiantes de 15 años escolarizados en cada CCAA, ya que algunos resultados se ofrecerán ponderados por este valor.

\section{Análisis de datos}

En relación al primer objetivo se calcularon descriptivos básicos y se analizó la distribución de frecuencias. Para el segundo objetivo se estimaron correlaciones de Pearson con pesos senatoriales y ponderados. En el primer caso todas las administraciones tenían el mismo peso, mientras que en el segundo las administraciones con mayor población de 15 años contribuyeron en mayor medida a la estimación. Para cubrir el último objetivo se realizó una comparación jerárquica de dos modelos de regresión múltiple por cada resultado en PISA 2015. Los predictores del Modelo 1 fueron los tres indicadores de recursos: gasto por estudiante, salario docente y ratio. El Modelo 2 mantiene la terna anterior y añade el PIB per cápita (riqueza) y la tasa de idoneidad a los 15 años. Los modelos se ajustaron con los pesos senatoriales y su potencia explicativa se comprobó con el estadístico $\mathrm{R}^{2}$ ajustado, ya que contienen distinto número predictores. Los análisis se realizaron con SPSS v.21.

\section{Resultados}

Distribución de recursos en las Administraciones Educativas

La Tabla 1 muestra que la riqueza y recursos no se reparten homogéneamente. Así, el PIB per cápita de Madrid prácticamente duplica el de Extremadura. Todas las comunidades con menos de $20.000 €$ per cápita se ubican en el sur peninsular, mientras que las regiones de la mitad norte presentan valores cercanos al promedio nacional (23.296€). La Comunidad Valenciana es la única excepción en esta lectura: ubicándose en la mitad sur tiene un PIB per cápita similar a las comunidades del noroeste cantábrico.

Tabla 1. Distribución de los indicadores de riqueza y recursos por CCAA

\begin{tabular}{|l|c|c|c|c|}
\cline { 2 - 5 } \multicolumn{1}{l|}{} & $\begin{array}{c}\text { PIB } x \\
\text { cápita }\end{array}$ & $\begin{array}{c}\text { Gasto } x \\
\text { estudiante }\end{array}$ & $\begin{array}{c}\text { Salario } \\
\text { docente }\end{array}$ & Ratio \\
\hline Andalucía & 17356 & 4220 & 31410 & 13,1 \\
\hline Aragón & 25214 & 4891 & 31302 & 12,1 \\
\hline Asturias & 20351 & 5747 & 32963 & 10,9 \\
\hline Baleares & 24446 & 5102 & 34669 & 11,7 \\
\hline Canarias & 19340 & 4627 & 34103 & 13,7 \\
\hline Cantabria & 20875 & 5644 & 33781 & 11,2 \\
\hline Castilla y León & 21723 & 5372 & 31642 & 11,3 \\
\hline Castilla-La Mancha & 18229 & 4545 & 33182 & 12,4 \\
\hline Cataluña & 27765 & 4580 & 31511 & 13,2 \\
\hline Comunidad Valenciana & 20451 & 4628 & 31823 & 12,9 \\
\hline Extremadura & 16111 & 5595 & 31591 & 11,3 \\
\hline Galicia & 20660 & 5585 & 31053 & 10,5 \\
\hline Madrid & 31917 & 3957 & 31333 & 14,1 \\
\hline Murcia & 19287 & 4545 & 32638 & 12,5 \\
\hline Navarra & 28925 & 5731 & 33067 & 11,3 \\
\hline País Vasco & 30568 & 6437 & 39619 & 12,1 \\
\hline Rioja, La & 25209 & 5004 & 32918 & 12,3 \\
\hline Ceuta & 18938 & 4988 & 41220 & 13,7 \\
\hline Melilla & 17219 & 5003 & 41220 & 13,7 \\
\hline Promedio Espana & 23296 & 4743 & 34008 & 12,7 \\
\hline & & & & \\
\hline
\end{tabular}

El gasto por estudiante también presenta importantes variaciones regionales: el País Vasco se diferencia del resto, y supera en un $63 \%$ a Madrid, la administración con el valor más bajo. Las CCAA se pueden asignar a tres grupos: en el primero están aquellas cuyo gasto por estudiante se encuentra sobre los $5.500 €$. Todas ellas, salvo Extremadura, de la mitad norte peninsular. El segundo grupo está conformado por administraciones en torno a $5.000 €$ y el último por las comunidades cuyo gasto por estudiante ronda los $4.500 €$. En este último grupo se encuentran las regiones del mediterráneo peninsular además de Canarias y Castilla La Mancha. Es destacable que hay comunidades con un alto PIB per cápita (Madrid, Aragón y Cataluña) cuyo gasto por estudiante es in- 
Tabla 2. Distribución de los indicadores de resultados educativos por CCAA

\begin{tabular}{|c|c|c|c|c|}
\hline & $\begin{array}{l}\text { Tasa de idoneidad } \\
\text { a los } 15 \text { años }\end{array}$ & $\begin{array}{c}\text { Media PISA-2015 } \\
\text { Lectura }\end{array}$ & $\begin{array}{l}\text { Media PISA-2015 } \\
\text { Matemáticas }\end{array}$ & $\begin{array}{c}\text { Media PISA-2015 } \\
\text { Ciencias }\end{array}$ \\
\hline Andalucía & 63,0 & 479 & 466 & 473 \\
\hline Aragón & 54,0 & 506 & 500 & 508 \\
\hline Asturias & 69,0 & 498 & 492 & 501 \\
\hline Baleares & 61,0 & 485 & 493 & 485 \\
\hline Canarias & 60,0 & 483 & 452 & 475 \\
\hline Cantabria & 67,0 & 501 & 495 & 496 \\
\hline Castilla y León & 64,0 & 522 & 506 & 519 \\
\hline Castilla La Mancha & 59,0 & 499 & 486 & 497 \\
\hline Cataluña & 77,0 & 500 & 500 & 504 \\
\hline Comunidad Valenciana & 62,0 & 499 & 485 & 494 \\
\hline Extremadura & 64,0 & 475 & 473 & 474 \\
\hline Galicia & 67,0 & 509 & 494 & 512 \\
\hline Madrid & 67,0 & 520 & 503 & 516 \\
\hline Murcia & 60,0 & 486 & 470 & 484 \\
\hline Navarra & 73,0 & 514 & 518 & 512 \\
\hline País Vasco & 74,0 & 491 & 492 & 483 \\
\hline Rioja, La & 66,0 & 491 & 505 & 498 \\
\hline Ceuta & 52,0 & - & - & - \\
\hline Melilla & 56,0 & - & - & - \\
\hline Promedio España & 65,8 & 496 & 486 & 493 \\
\hline
\end{tabular}

Nota: El Ministerio de Educación no publicó resultados separados para Ceuta y Melilla

ferior a la media nacional. En cambio, otras comunidades con un PIB per cápita inferior a la media (Galicia, Extremadura, Castilla y León, Cantabria y Asturias) tienen un gasto por estudiante superior al promedio. Por tanto, las CCAA también presentan fuertes variaciones en la porción de riqueza destinada al gasto educativo.

Exceptuando el País Vasco, el salario anual bruto del profesorado es más elevado en las administraciones insulares y del norte de África debido al complemento de destino extra-peninsular. En el resto el rango salarial oscila entre $33.067 €$ (Navarra) y $31.053 €$ (Galicia).

En el caso de la ratio estudiantes/docente nuevamente se cumple la regla de que, exceptuando Extremadura, las administraciones del norte peninsular presentan las ratios más bajas. Además, se observa que las administraciones con mayor gasto por estudiante tienden a presentan ratios más bajas.

\section{Resultados educativos en las Administraciones educativas}

La Tabla 2 recoge la distribución de los cuatro indicadores de resultados. La tasa de idoneidad señala que, en el conjunto de España, prácticamente 1 de cada 3 estudiantes acumula retraso antes de finalizar los estudios obligatorios. De nuevo, se observan grandes diferencias: en algunas regiones la tasa de idoneidad está en torno al $75 \%$, mientras que en otras prácticamente la mitad del alumnado repite durante la escolarización obligatoria.

Los promedios en PISA 2015 también señalan importantes variaciones. Sirva como criterio de comparación que la OECD (2016b) estima que aproximadamente 40 puntos en la escala PISA equivalen a un curso escolar completo. En el conjunto de la prueba Castilla y León, Madrid y Navarra presentan los mejores resultados, mientras que Canarias, Extremadura y Andalucía se encuentran en el extremo contrario.

\section{Relación entre recursos y resultados educativos}

La Tabla 3 muestra las correlaciones de Pearson, que en el triángulo superior están ponderadas $\left(\mathrm{r}_{\mathrm{w}}\right)$ por el número de estudiantes de 15 años de cada CCAA y en el inferior emplean pesos senatoriales $\left(\mathrm{r}_{\mathrm{nw}}\right)$. En primer lugar, se comentan las correlaciones entre las variables de recursos; a continuación, las correlaciones entre los indicadores de resultados; y finalmente, los 16 pares producto de cruzar variables de recursos y resultados.

Entre las variables de recursos las correlaciones de mayor magnitud corresponden al gasto por estudiante, que presenta una asociación alta y positiva con el salario docente, especialmente con los datos ponderados $\left(\mathrm{r}_{\mathrm{w}}=, 61\right)$, y muy alta y negativa con la ratio estudiantes/docente $\left(r_{w}=-, 81\right.$ y $\left.r_{n w}=-, 72\right)$. En el resto de variables de recursos las correlaciones son muy débiles o arrojan resultados poco concluyentes.

Lógicamente, las correlaciones entre las puntuaciones en PISA son muy altas, mientras que la tasa de idoneidad muestra asociaciones positivas con los resultados en PISA que oscilan entre bajas y moderadas, lo que permite afirmar que las regiones con 
Tabla 3. Correlaciones de Pearson entre las variables

\begin{tabular}{|l|c|c|c|c|c|c|c|c|}
\cline { 2 - 9 } \multicolumn{1}{l|}{} & 1 & 2 & 3 & 4 & 5 & 6 & 7 & 8 \\
\hline 1. PIB x cápita & -- &,- 03 &, 12 &, 35 &, 62 &, 69 &, 78 &, 69 \\
\hline 2. Gasto x estudiante &, 11 & -- &, 61 &,- 81 &, 18 &, 00 &, 20 &, 04 \\
\hline 3. Salario docente &,- 08 &, 29 & -- &,- 17 &, 05 &,- 20 &,- 07 &,- 26 \\
\hline 4. Ratio &, 04 &,- 72 &, 34 & -- &, 09 &, 03 &,- 12 &,- 05 \\
\hline 5. Tasa de idoneidad a los 15 años &, 58 &, 38 &,- 29 &,- 33 & -- &, 25 &, 53 &, 35 \\
\hline 6. PISA-2015 Lectura &, 50 &, 03 &,- 27 &,- 13 &, 22 & -- &, 85 &, 97 \\
\hline 7. PISA-2015 Matemáticas &, 68 &, 30 &,- 05 &,- 35 &, 44 &, 75 & -- &, 91 \\
\hline 8. PISA-2015 Ciencias &, 49 &, 04 &,- 37 &,- 22 &, 23 &, 96 &, 83 & - \\
\hline
\end{tabular}

Nota: Triángulo superior: correlaciones estimadas con pesos ponderados $\left(\mathrm{r}_{\mathrm{w}}\right)$; Triángulo inferior: con pesos senatoriales ( $\left.\mathrm{r}_{\mathrm{nw}}\right)$

mayor porcentaje de repetición tienden a mostrar resultados más bajos en PISA 2015.

En el cruce de variables de recursos y resultados la principal conclusión es que las administraciones más ricas tienden a presentar promedios en PISA más altos y tasas de repetición más bajas. En general, los datos ponderados ofrecen magnitudes ligeramente superiores a los pesos senatoriales. En el resto de pares de variables las magnitudes de las asociaciones son bajas o muy bajas.

\section{Ejemplos gráficos de los análisis de correlaciones}

En este apartado se ejemplificará el significado de las magnitudes de los coeficientes de correlaciones de la Tabla 3. En las figuras se han empleado datos ponderados, por lo que el tamaño de los puntos es proporcional al número de estudiantes de 15 años escolarizados en cada región.

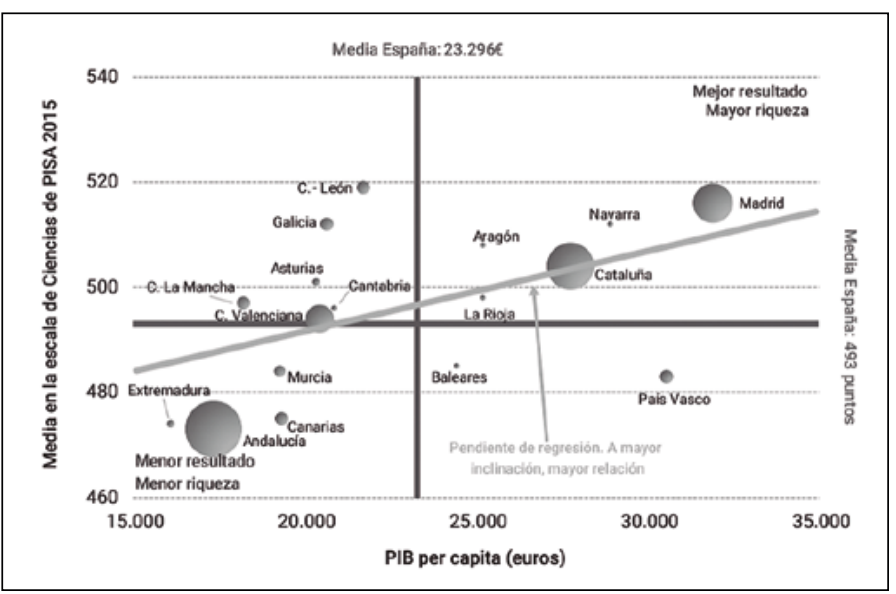

Fuente: Servicio de Evaluación Educativa de la Consejería de Educación y Cultura del Gobierno del Principado de Asturias (2019).

Figura 1. Relación entre Resultados en Ciencias y PIB per cápita

La Figura 1 ilustra la relación entre resultados y riqueza de las CCAA, tomando como muestra los resultados en Ciencias y el PIB per cápita, dos variables fuertemente asociadas $\left(\mathrm{r}_{\mathrm{w}}=, 69\right)$. La magnitud de la asociación está representada por la pendiente de regresión: su inclinación señala que las CCAA más ricas tienden a presentar mejores resultados. No obstante, aunque la relación es fuerte no determina los resultados completamente. Las CCAA que se sitúan por encima de la pendiente de regresión (v. g., Castilla y León, Galicia, Castilla-La Mancha o Asturias) obtienen resultados superiores a lo esperado por su nivel de riqueza. Por el contrario, los puntos situados por debajo de la pendiente señalan regiones cuyo promedio en Ciencias es inferior a lo esperado por su riqueza.

La Figura 2 ejemplifica la relación entre el gasto público por estudiante y los resultados escolares, los cuales están representados por la puntuación en Ciencias. En este caso la recta de regresión es prácticamente plana, indicando ausencia de relación entre ambas variables $\left(r_{w}=, 04\right)$. Las regiones situadas a la derecha tienen un mayor gasto por estudiante, pero sus promedios en Ciencias son muy dispares. Similar lectura se realiza para las regiones con menor gasto por estudiante.

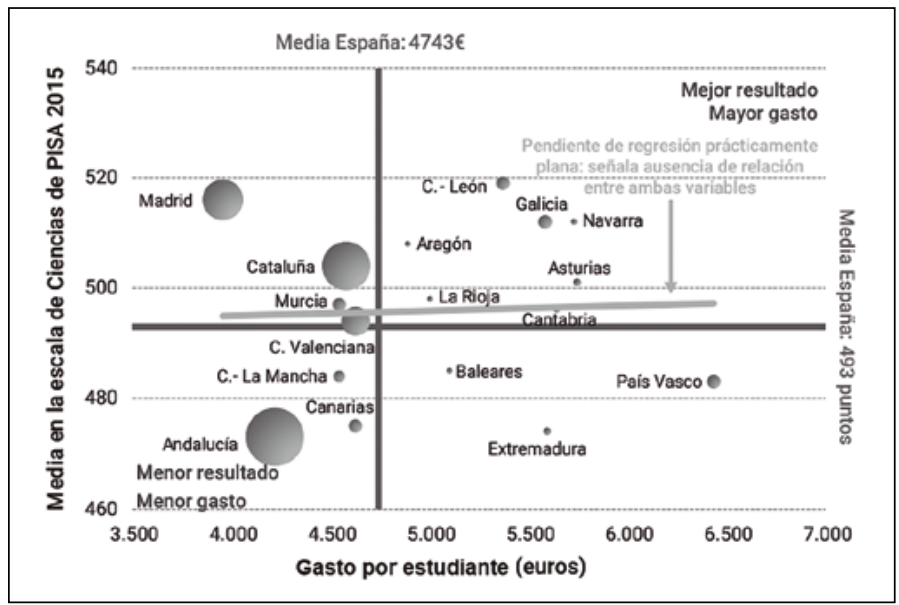

Fuente Servicio de Evaluación Educativa de la Consejería de Educación y Cultura del Gobierno del Principado de Asturias.

Figura 2. Relación entre Resultados en Ciencias y Gasto por estudiante

La Figura 3 representa la relación entre salario docente y resultados en Matemáticas. De nuevo, la línea de regresión es prácticamente plana, incluso descendente $\left(r_{w}=-, 07\right)$ lo que señala que los promedios de las CCAA en PISA 2015 no guardan relación con el salario de sus docentes. 


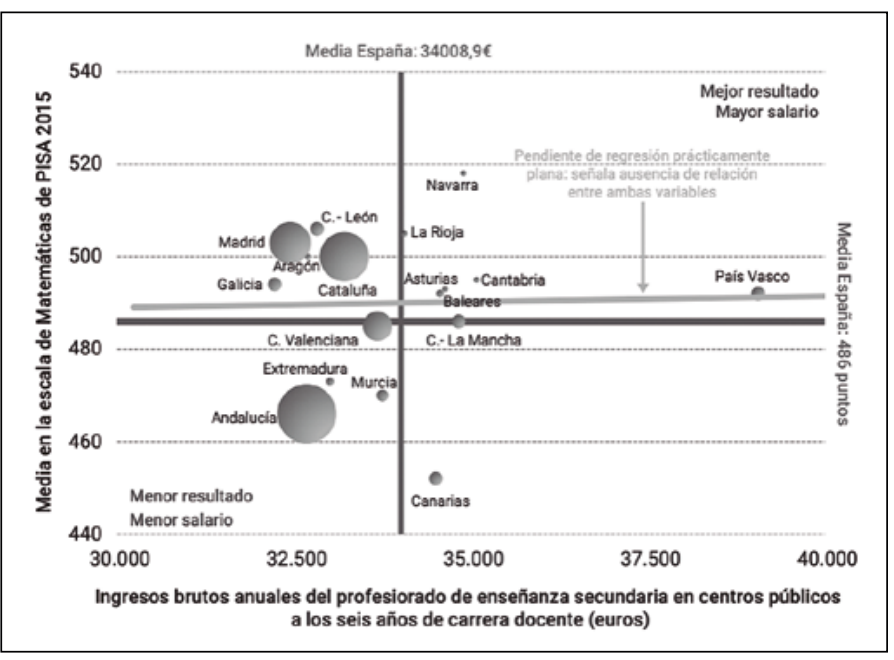

Fuente Servicio de Evaluación Educativa de la Consejería de Educación y Cultura del Gobierno del Principado de Asturias (2019).

Figura 3. Relación entre los Resultados en Matemáticas

$$
\text { y Salario bruto docente }
$$

La Figura 4 ejemplifica la relación entre la ratio estudiantes/ docente y los resultados escolares, representados en este caso por la tasa de idoneidad a los 15 años. Los datos indican nuevamen-

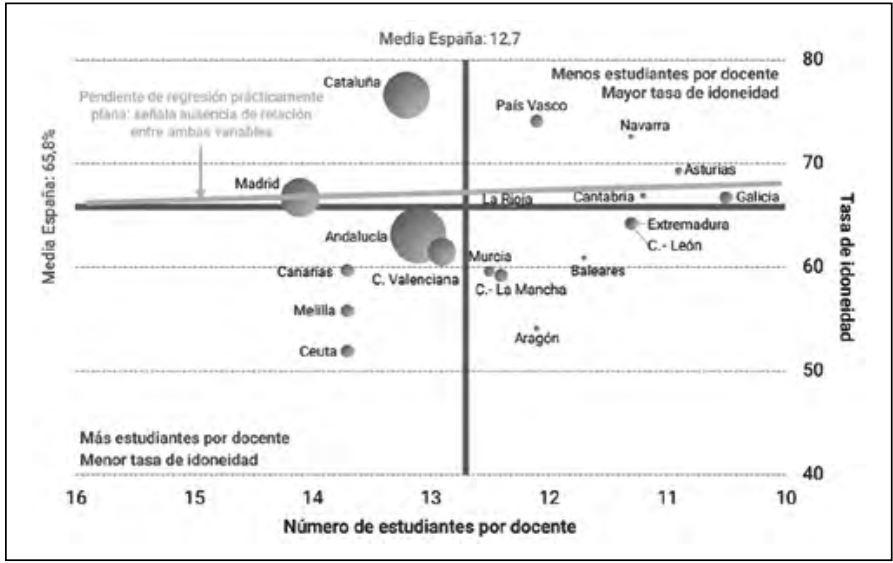

Fuente. Servicio de Evaluación Educativa de la Consejería de Educación y Cultura del Gobierno del Principado de Asturias (2019).

Figura 4. Relación entre Tasa de idoneidad a los 15 años y Ratio estudiantes/docente

te una relación casi nula $\left(\mathrm{r}_{\mathrm{w}}=, 09\right)$ : el número de estudiantes por docente parece influir muy poco en las tasas de repetición de las regiones.

Tabla 4. Modelos de regresión para predecir los resultados en PISA 2015

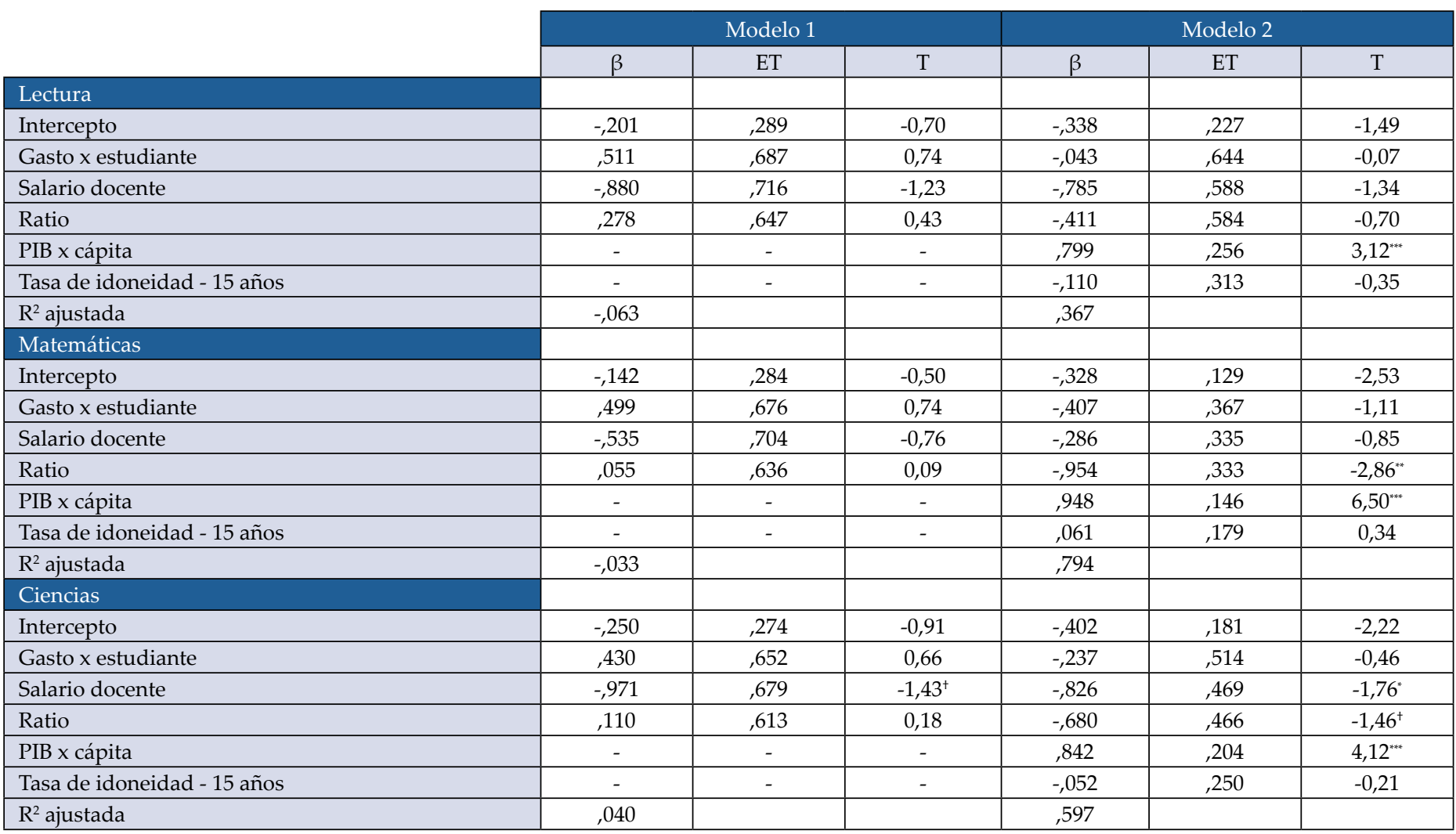

$\beta$ : Coeficiente estandarizado de regresión.

ET: Error típico del coeficiente.

T: Valor del estadístico t para determinar niveles de significación. Dado que el conjunto de datos es pequeño ( $\mathrm{N}=17)$ se han señalado los valores $t$ que señalan niveles marginalmente significativos $(\alpha<0,10$ y $\alpha<0,20)$.

Niveles de significación: ${ }^{* * *}: \alpha<0,01 ;{ }^{* *}: \alpha<0,05 ;{ }^{*}: \alpha<0,10 ;{ }^{+}: \alpha<0,20$. 
Modelos de regresión múltiple para predecir los resultados en PISA

La Tabla 4 muestra los resultados de los modelos de regresión para predecir los resultados de las comunidades en las tres materias evaluadas PISA 2015. La capacidad predictiva del Modelo 1 es muy baja. De hecho, el valor de $\mathrm{R}^{2}$ ajustado es prácticamente nulo en las tres materias, y en dos casos incluso de signo negativo lo que confirma que los tres indicadores del Modelo 1 no son buenos predictores de los resultados regionales en PISA. En este modelo sólo el salario docente presenta una significación estadística marginal $(\alpha<0,20)$ en el caso de Ciencias, si bien el coeficiente de regresión es negativo y su capacidad explicativa muy limitada.

Por su parte, el Modelo 2 tiene una mayor potencia predictiva: $\mathrm{R}^{2}$ ajustado oscila entre ,367 (Lectura) y ,794 (Matemáticas). Sin duda, el incremento del coeficiente de determinación es imputable a la inclusión del PIB per cápita, que es la única variable con clara significación estadística en las tres materias. En el conjunto de los Modelos 2 también es interesante el efecto de la Ratio, toda vez que aparece una relación inversa entre esta variable y los resultados Matemáticas y Ciencias, si bien en este último caso la significación es marginal.

\section{Discusión}

Por su naturaleza pública, la inversión en educación es un campo abonado al debate social: es relativamente sencillo encontrar reseñas donde familias, docentes, agentes sociales y responsables educativos señalan la escasez de recursos como una de las causas de los bajos resultados educativos y un limitante del derecho a la educación (González, 2018). Este escrito pretende aportar evidencias para el debate analizando la distribución de los recursos y la inversión educativa en España, y estudiando la conexión entre los indicadores de recursos y resultados en PISA 2015.

En relación al primer objetivo se observa que, en España los niveles de riqueza e inversión educativa no se distribuyen homogéneamente. En general las comunidades del norte peninsular presentan mayor gasto por estudiante y ratios más bajas que las comunidades de la mitad sur. En el caso del ingreso bruto docente no se cumple este patrón ya que los salarios más altos corresponden a los territorios extra-peninsulares (además del País Vasco). En todo caso, la riqueza regional parece poco relacionada con el nivel de inversión educativa ya que existen importantes diferencias en el porcentaje del PIB per cápita que cada administración destina al gasto por estudiante. Igualmente, también existen claras diferencias regionales en los indicadores de resultados, volviendo a destacar las CCAA de la mitad norte que se sitúan sobre el promedio de la OECD y, en el mejor de los casos, alcanzan puntuaciones similares a Corea del Sur en matemáticas y ciencias y Finlandia en lectura (OECD, 2016b). Análogamente, la tasa de idoneidad a los 15 años señala que los índices de repetición son más altos en las comunidades del sur peninsular, las islas y Ceuta y Melilla. Por tanto, las regiones con mejores resultados en PISA tienden a presentar tasas de repetición más bajas, hecho que es coherente con los resultados internacionales de PISA (Ikeda y García, 2014).

Con respecto al segundo objetivo del estudio se observa que las correlaciones entre los indicadores de rendimiento y de recursos e inversión son, en general, muy bajas, evidencia compatible con gran parte de la investigación previa (Glewwe y Lambert, 2010; Glewwe et al., 2015; Jackson et al., 2016; Leuven y Oosterbeek, 2018; OECD, 2016a). La explicación a estos datos parece radicar en el hecho de que España se sitúa en umbrales de renta y gasto por estudiante donde la relación entre inversión y resultados escolares se diluye (Arroyo-Resino et al., 2019; Vegas y Coffin, 2015). De hecho, las diferencias en el gasto educativo entre comunidades con resultados PISA similares reafirma la idea de que el aumento del gasto en países desarrollados tiene un impacto pequeño en los logros académicos (Glewwe y Lambert, 2010).
Finalmente, los modelos de regresión ajustados señalan que el PIB per cápita es la única variable capaz de predecir de forma consistente y significativa los resultados en PISA de las CCAA. En general, las regiones con un nivel de renta más alto presentan mejores resultados. Por su parte, los indicadores de recursos apenas presentan significaciones estadísticas y cuando lo hacen son magnitudes marginales y poco sistemáticas. Estos datos son compatibles las evidencias previas que señalan que, en los países más desarrollados la relación entre indicadores de recursos y resultados escolares es débil y poco consistente (Arroyo-Resino et al., 2019; Coffield, 2012; Glewwe y Lambert, 2010; Hanushek, 1986, 1997; Jackson et al., 2016; Leuven y Oosterbeek, 2018; OECD, 2016a; Pritchett y Filmer, 1999; Vegas y Coffin, 2015).

Esta investigación presenta algunas limitaciones. En primer lugar, es un estudio correlacional, con una única medida transversal y un número limitado de casos. Por tanto, los resultados no pueden interpretarse en términos causales y, en el futuro, sería necesario extender el estudio a otros cursos o etapas, y establecer un diseño que permita el análisis de medidas repetidas. Además, los resultados se circunscriben a un contexto concreto: un país relativamente rico, alto consumidor educativo $\mathrm{y}$, al tiempo, con una política educativa descentralizada. Todo ello dificulta extrapolar las conclusiones a otros contextos culturales.

\section{Conclusiones}

Respondiendo a la pregunta que daba título a este escrito parece que una oferta educativa de calidad necesita dos condiciones básicas: un contexto económico con cierta holgura, es decir, una renta per cápita esté por encima de 20.000 \$-PPA y una inversión acumulada por estudiante entre los 6 y los 15 años superior a 35.000 \$-PPA (OECD, 2012). Si estas cantidades se transformaran a euros del año 2015 para un país con el poder adquisitivo de España supondrían algo más de $13.000 €$ de renta per cápita y casi $23.000 €$ de gasto acumulado por estudiante. Alcanzados dichos umbrales los modelos predictivos que conectan los indicadores de inversión y recursos con los resultados escolares arrojan relaciones débiles y poco consistentes. España cumple las dos condiciones mencionadas, tal y como reflejan los datos de la Tabla 1. Probablemente por ello en el presente análisis los indicadores de inversión y recursos no parecen ser buenos predictores de los resultados en PISA de las CCAA. La variable que mejor explica las diferencias es el nivel de riqueza de las regiones. Todo ello es coherente con las conclusiones de la investigación que señalan que, alcanzado cierto nivel de gasto e inversión lo que marca las posibilidades de éxito escolar no es el volumen total de gatos sino la gestión de esa inversión (Glewwe et al., 2015; Britton y Vignoles, 2017; Card y Krueger, 1996; Gamoran y Long, 2007). Esta última afirmación tiene importantes implicaciones educativas: las comparaciones internacionales (OECD, 2010) advierten que, independientemente de la riqueza y el volumen de gasto, los sistemas educativos con mejores resultados orientan sus recursos al éxito educativo para todos: establecen políticas educativas inclusivas, limitan la medida de repetición escolar y no permiten la transferencia a otros centros del alumnado con dificultades de aprendizaje, ni la creación de grupos por niveles de rendimiento, problemas de comportamiento o necesidades educativas dentro de los centros.

\section{Agradecimientos}

Esta investigación fue financiada por la Universidad de Oviedo. Referencia: FUO-18-262.

\section{Contribución individual de los autores y autoras al manuscrito}

JML ha participado en la conceptualización, gestión de la base de datos, análisis de los datos, metodología y escrito del borrador original. RFA ha participado en la conceptualización, meto- 
dología, supervisión, revisión del escrito, edición y adquisición de la financiación.

\section{Referencias}

Abott, C., Kogan, V., Lavertu, S. y Peskowitz, Z. (2020). School district operational spending and student outcomes: Evidence from tax elections in seven states. Journal of Public Economics, 183, 104-142. https://doi.org/10.1016/j.jpubeco.2020.104142

Arroyo-Resino, D., Constante-Amores, I. y Asensio-Muñoz, I. (2019). La repetición de curso a debate: un estudio empírico a partir de PISA 2015. Educación XX1, 22(2), 69-92. https://doi. org/10.5944/educxx1.22479

Britton, J. y Vignoles, A. (2017). Education production functions. En G. Johnes, J. Johnes, T., Agasisti y L. López-Torres (Eds.), Handbook of Contemporary Education Economics (pp. 246-271). Edward Elgar Publishing Ltd. https://doi. org/10.4337/9781785369070.00016

Candelaria, C. y Shores, K. (2019). Court-ordered finance reforms in the adequacy ERA: Heterogeneous causal effects and sensitivity. Education Finance and Policy 14(1), 31-60. https://doi. org/10.1162/edfp_a_00236

Card, D. y Krueger, A. (1996). School resources and student outcomes: An overview of the literature and new evidence from North and South Carolina. Journal of Economic Perspectives, 10(4), 31-50. https://doi.org/10.1257/jep.10.4.31

Coffield, F. (2012). Why the McKinsey reports will not improve school systems. Journal of Education Policy, 27(1), 131-149. https://doi.org/10.1080/02680939.2011.623243

Cunningham, C., Cunningham, S., Halim, N. y Yount, K. (2019). Public investments in education and children's academic achievements. Journal of Development Studies, 55(11), 23652381. https://doi.org/10.1080/00220388.2018.1516869

Fernández-Alonso, R. y Muñiz, J. (2019). Calidad de los sistemas educativos: Modelos de evaluación. Propósitos y Representaciones, 7(spe), e347. https://dx.doi.org/10.20511/pyr2019.v7nSPE.347

Filges, T., Sonne-Schmidt, C. y Nielsen, B. (2018). Small class sizes for improving student achievement in primary and secondary schools: A systematic review. Campbell Systematic Reviews, 14(1), 1-107. https://doi.org/10.4073/csr.2018.10

Gamoran, A. y Long, D. (2007). Equality of educational opportunity a 40 year retrospective. En R. Teese, S. Lamb, M. Duru-Bellat y S. Helme (Eds.), International Studies in Educational Inequality, Theory and Policy (pp. 23-47). Springer. https://doi. org/10.1007/978-1-4020-5916-2_2

Gaviria, J., Martínez-Arias, R. y Castro, M. (2004). Un estudio multinivel sobre los factores de eficacia escolar en países en desarrollo: El caso de los recursos en Brasil. Education Policy Analysis Archives, 12(20), 1-31. https://epaa.asu.edu/ojs/article/ viewFile/175/301

Glewwe, P., Hanushek, E., Humpage, S. y Ravina, R. (2015). School resources and educational outcomes in developing countries: A review of the literature from 1990 to 2010. En P. Glewwe (Ed.), Education Policy in Developing Countries (pp. 13-64). Chicago Scholarship Online. https://doi.org/10.7208/ chicago/9780226078854.003.0002

Glewwe, P. y Lambert, S. (2010). Education production functions: Evidence from developing countries. En P. Peterson, E. Baker y B. McGaw (Eds.), International Encyclopedia of Education (3 $3^{\mathrm{a}}$ ed., pp. 412-422). Elsevier Ltd. https://doi.org/10.1016/B978-008-044894-7.01232-X

González Álvarez, M. (2017). Algunas consideraciones en relación con el derecho a la educación en la comunidad autónoma de Asturias. Magister, 29(1), 17-24 https://doi.org/10.17811/ msg.29.1.2017.17-24
Hanushek, E. (1986). The economics of schooling: Production and efficiency in public schools. Journal of Economic Literature, 49(3), 1141-1177. https://www.jstor.org/stable/2725865

Hanushek, E. (1997). Assessing the effects of school resources on student performance: An update. Educational Evaluation and Policy Analysis, 19(2), 141-164. https://doi. org/10.3102/01623737019002141

Hanushek, E., Piopiunik, M. y Wiederhold, S. (2019). The value of smarter teachers. Journal of Human Resources, 54(4), 857-899. https://doi.org/10.3368/jhr.54.4.0317.8619r1

Hanushek, E. y Woessmann, L. (2011). The economics of international differences in educational achievement. En E. A. Hanushek, S. Machin y L. Woessmann (Eds.), Handbook of the Economics of Education (Vol. 3, pp. 89-200). Elsevier. https://doi. org/10.1016/B978-0-444-53444-6.00004-3

Harbison, R. y Hanushek, E. (1992). Educational performance of the poor: lessons from rural northeast Brazil. Oxford University Press. https://doi.org/10.1016/0738-0593(93)90051-z

Ikeda, M. y García E. (2014). Grade repetition: A comparative study of academic and non-academic consequences. OECD Journal: Economic Studies, (2013)1. http://dx.doi.org/10.1787/ eco_studies-2013-5k3w65mx3hnx

Jackson, C. K., Johnson, R. C. y Persico C. (2016). The effects of school spending on educational and economic outcomes: Evidence from school finance reforms. The Quarterly Journal of Economics, 131(1), 157-218. https://doi.org/10.1093/qje/qjv036.

Krueger, A. B. (1999). Experimental estimates of education production functions. The Quarterly Journal of Economics, 114(2), 497-532. https://doi.org/10.1162/003355399556052

Lafortune, J., Rothstein, J. y Schanzenbach, D. (2018). School finance reform and the distribution of student achievement. American Economic Journal: Applied Economics, 10(2), 1-26. https:// doi.org/10.1257/app.20160567

Leclercq, F. (2005). The relationship between educational expenditures and outcomes. Document de Travail DT/2005-05. Développement Institutions et Analyses à Long Terme (DIAL)/ UNESCO https://dial.ird.fr/content/download/49283/378447/ version/1/file/2005-05.pdf

Leuven, E. y Oosterbeek, H. (2018). Class size and student outcomes in Europe. European Expert Network on Economics of Education Analytical Report, 33. Publications Office of the European Union https://doi.org/10.2766/59709

Molnar, A., Smith, P., Zahorik, J., Halbach, A., Ehrle, K., Hoffman, L. y Cross, B. (2001). Evaluation results of the Student Achievement Guarantee in Education (SAGE) Program. University Of Wisconsin-Milwaukee. https://nepc.info/sites/default/files/ epru-0201-104.pdf

OECD. (2010). PISA 2009 results: What makes a school successful? Resources, policies and practices (Volume IV). OECD Publishing. https://doi.org/10.1787/9789264091559-en

OECD. (2012). Equity and quality in education: supporting disadvantaged students and schools. OECD Publishing. http://dx.doi.org/10.1787/9789264130852-en.

OECD. (2016a). Education at a glance 2016: OECD Indicators. OECD Publishing. https://doi.org/10.1787/eag-2016-en.

OECD. (2016b). PISA 2015 results (Volume I): Excellence and equity in education. OECD Publishing. https://doi.org/10.1787/9789264266490-en.

Pritchett, L. y Filmer, D. (1999). What education production functions really show: A positive theory of education expenditures. Economics of Education Review, (18)2, 223-239. https://doi. org/10.1016/s0272-7757(98)00034-x

Rivkin, S., Hanushek, E. y Kain, J. (2005). Teachers, schools, and academic achievement. Econometrica, 73(2), 417-458. https:// doi.org/10.1111/j.1468-0262.2005.00584.x

Schwerdt, G. y Woessmann, L. (2020). Empirical methods in the economics of education. En S. Bradley y C. Green (Eds.), The Economics of Education: A Comprehensive Overview (pp. 3-20). 
Academic Press. https://doi.org/10.1016/B978-0-12-8153918.00001-X

Servicio de Evaluación Educativa de la Consejería de Educación y Cultura del Gobierno del Principado de Asturias (2019) ¿Cuánto importa el gasto educativo? Informes de Evaluación, 19. https://www.educastur.es/-/informe-evaluacion-n-19-cuanto-importa-el-gasto-educativo

United Nations. (2015). General Assembly Resolution A/RES/70/1. Transforming Our World, the 2030 Agenda for Sustainable Development. https://undocs.org/A/RES/70/1
Vegas, E. y Coffin, C. (2015). When education expenditure matters: An empirical analysis of recent international data. Comparative Education Review, 59(2), 289-304. https://doi.org/10.1086/680324

Zyngier, D. (2014). Class size and academic results, with a focus on children from culturally, linguistically and economically disenfranchised communities. Evidence Base, 2014(1), 1-24. https://doi.org/10.21307/eb-2014-001 